\title{
Hasta que la muerte las separe Crítica literaria y teoría en la Argentina (algunas notas) ${ }^{1}$
}

MIGUEL DALMARONI Universidad Nacional de La Plata - CONICET, Argentina / dalmaroni@gmail.com

\section{Resumen}

Se ensayan aquí tres hipótesis: la estrecha y duradera relación de la crítica literaria argentina con la teoría no es una característica local (aunque no universal, es más bien global); en la Argentina la teoría literaria se enseña pero no se escribe; «teoría» es un nombre reemplazable, que usábamos en el siglo XX para señalar de modo tentativo un tipo de pensamiento acerca de ciertas contingencias donde interviene algo que seguimos llamando «literatura».

Palabras clave: crítica literaria argentina / teoría literaria / América Latina / universidad argentina

\section{Till death them do part. Literary criticism and theory in Argentina (some notes) \\ Abstract}

Three hypotheses are explored here: the close and long-lasting relationship between Argentine literary criticism and theory is not a local feature (although not universal, it is rather global); in Argentina, literary theory is taught but not written; «theory» is a replaceable name, which back in the 1900s we used in a tentative way to refer to a kind of thinking about certain contingencies where something that we still call «literature» is involved.

Key words: argentine literary criticism / literary theory / Latin America / argentine university

Recibido: 12/9/2018. Aceptado: 15/10/2018

Para citar este artículo: Dalmaroni, Miguel (2018). Hasta que la muerte nos separe. Crítica literaria y teoría en la Argentina (algunas notas). El taco en la brea, 8 (junio-noviembre), 101-109. Santa Fe, Argentina: UNL. DOI: $10.14409 /$ tb.v1i8.7759 


\section{Teoría es lo que se enseña}

En la Argentina, la teoría y la crítica literaria han estado unidas por un lazo matrimonial. Es más, un matrimonio por iglesia. La crítica es la amante y es criolla, la teoría es la amada (tiene ascendientes rusos pero es bastante francesa, algo alemana, algo británica; digamos con pasaporte de la Comunidad Europea, incluso después de haber ensayado un par de mudanzas aquende el Atlántico).

La figura, obviamente, es poco seria y, en tren de divertimentos podríamos ir todo lo lejos que fuese posible: fue un matrimonio arreglado entre los patriotas de mayo y la Generación del 37. Y así. Poco seria y exagerada, pero ¿cuán exagerada es la figura? Si la redujésemos a argumentos, habría dos: el pensamiento escrito sobre literatura en la Argentina es crítica literaria argentina, no teoría; la relación entre las dos es duradera, intensa y conflictiva, y posiblemente constitutiva.

Aunque el curso mismo de estas notas persigue enfatizarlo, el segundo argumento —el que responde a la figura matrimonial - sin dudas es tan contrastable y sabido como inespecífico: sería un exceso de provincianismo, una pretensión de localía con tanta base como la de cualquier otro país, dar por hecho que ese vínculo vitalicio e intenso entre crítica y teoría es un rasgo argentino (o, peor, una rareza local); por supuesto que con tonos e historias variadas, sucede lo mismo en muchas otras regiones, circuitos y zonas de las comunidades y corporaciones críticas. Cuando en 2014 y en esta misma revista Graciela Montaldo procura caracterizar el estatuto actual de la teoría, de sus relaciones con la crítica, y de la historia de esas relaciones en la Argentina desde mediados del siglo XX, lo que describe es una situación que - en sus notas principales - se presenta no solo en América Latina: es una condición histórica de la crítica literaria. Pero volvamos al primer argumento: Susana Zanetti me contó hará algo más de veinte años que había no recuerdo en qué universidad cierto remanente o subsidio disponible, y ella y Carlos Altamirano tenían, parece, la posibilidad de indicar cómo gastarlo, invitando no solo a los latinoamericanos de siempre sino además a algún personaje nordhemisférico renombrado que lustrase con su sólida presencia teórica un coloquio más o menos selecto. Uno de los dos dijo «Starobinski. Podríamos traerlo a [Jean] Starobinski». Y recibió del otro —el tono ha de haber sido entre compasivo y socarrón pero la crónica ha perdido las circunstancias y no quiero inventar lo que no sé- esta réplica: «Y a Starobinski... ¿de qué le hablaríamos? ¿Del Martín Fierro?». No estoy seguro de si la anécdota podría depurarse de eurocentrismo y de machismo reemplazando a Starobinski por alguien como Gayatri Spivak, de quien podemos suponer, más o menos como de Starobinski, que acaso conociese, a lo más, algunos cuentos de Borges. Por supuesto, fuera del mundo hispanoamericano, incontables profesores universitarios de literatura han leído a Borges... pero solo han leído como teoría — solo han leído del mismo modo que leen el resto de lo que leen como teoría - lo que Harold Bloom, George Steiner, Foucault o Jacques Rancière escribieron sobre Borges. ¿O acaso alguien se imagina a Spivak o a Butler leyendo Borges, un escritor en las orillas de Sarlo como si leyese teoría literaria, es decir como nosotros hemos leído por décadas los ensayos de Bajtín sobre Dostoievski o los de Benjamin sobre Proust?

En la Universidad argentina los profesores de teoría literaria investigan y escriben sobre problemas de historia crítica de la literatura. Aquí la teoría literaria no se escribe, solo se enseña. O eso que se identifica y nombra como teoría literaria es un saber que casi únicamente se enseña (casi, ya que también se traduce y edita). ${ }^{2}$ A la vez, quienes no escribimos teoría pero la enseñamos, escribimos en cambio, característica o predominantemente, sobre literatura argentina. O podríamos decir, si se prefiere: los ensayos escritos aquí sobre la narrativa de Aira o sobre la poesía de Padeletti 
en los que usamos o hasta inventamos profusamente argumentos teóricos sobre la novela o sobre el sujeto poético, no se leen como teorías de la novela ni de la lírica, ni menos son catalogados como tal en las bibliotecas, ni siquiera ingresan muy a menudo en la bibliografía de cursos de teoría literaria (es así en la Argentina, de modo que... ¿quién imaginaría a un checo, una coreana o un canadiense - por decir — leyendo Las vueltas de César Aira como un libro de teoría literaria, o a una especialista valenciana incluyendo su reedición en una colección de teoría de la narrativa?

\section{Sube conmigo amor americano}

Un breve desvío, con esta otra exageración: quienes en cambio enseñan y escriben sobre literatura latinoamericana, ni escriben teoría ni la enseñan; aunque desde las revueltas anti-eurocéntricas de los años noventa, no pocos de ellos pudieron haber postulado que lo que escriben cuando escriben sobre literatura y cultura latinoamericana es (o conlleva) algún modo latinoamericano de la teoría, de lo que en los contextos de la crítica literaria y cultural latinoamericana, y con sedicente pleno derecho y credenciales harto probadas ya, haría las veces de la teoría (u ocuparía un espacio como el de la teoría). ¿Es esta última una posición política que obedece a motivos principalmente políticos aunque se presente y se tome a menudo como posición epistemológica y metodológica? Es lo que parece: en principio, los estudios literarios y culturales de cualquier región, la crítica literaria de cualquier región, podría tomarse por teoría; sobre todo si se considera que, a la inversa, casi de cualquier corpus de teoría literaria puede decirse que en realidad es crítica literaria -crítica de literatura francesa, inglesa, europea-. Por supuesto, estas distinciones y taxonomías podrían considerarse anacrónicas e incluso reaccionarias tras la supresión política de la «teoría» que tantos profesores de literatura latinoamericana mimaron con retórica radicalizada al fogonear o adoptar los dispositivos institucionales de lo poscolonial, lo subalternista, lo posoccidental y lo decolonial, por mencionar los principales dialectos de ese linaje. Por supuesto, no es necesario todo ese recorrido únicamente para acordar que convendría dar por reemplazada «teoría» por otras nominaciones más o menos provisorias (escrituras críticas, pensamiento crítico, etc.), pero es dable recordar aquí que la impugnación, la jubilación anticipada o el sobreseimiento de «teoría» fue una de las consecuencias de ese recorrido politicista de la corporación universitaria que conocemos como crítica anti eurocentrista, posdisciplinaria y feminista (una micro-fracción profesional con sede más o menos imaginaria, más o menos efectiva, en algunos congresos internacionales y en una red de universidades de los Estados Unidos, algunas de América Latina, unas pocas de Europa; en fin, una serie de grupos de intermitente presencia declarativa en los circuitos de comunicación académica, la prensa especializada, las redes sociales y la web, aunque con escasa, incidental y en todo caso mediata vinculación efectiva con acciones disidentes en los territorios y sus organizaciones de base, o con políticas emancipatorias, fuesen estatales, partidarias, sindicales, sociocomunitarias, económicas, educativas, etc.). ${ }^{3}$ Por supuesto, también en la Argentina es casi sentido común universitario la idea de que la literatura latinoamericana misma ha escrito en su interior una teoría literaria. Sería difícil no prestar acuerdo a semejante proposición, del mismo modo que no es posible negar que en Baudelaire, en Flaubert o en T.S. Eliot hay sólidos, influyentes y originales trabajos, momentos o ideas de teoría literaria. Que una teoría sea eurocéntrica y otra pueda resultar lo opuesto es otra cosa.

A la vez, lo que la convocatoria para este dossier llamó «el afán teoricista del campo latinoamericano» es en efecto un tinte intenso de la crítica literaria y cultural del subcontinente, pero 
como anticipé, no creo que el afán teoricista sea un rasgo propio ni característico del campo latinoamericano. Tal cosa se confirma rápidamente si se repasan apenas las firmas más citadas en la crítica cultural y política que se produce y se estudia en USA y en parte de Europa desde hace unos 30 ó 40 años, especialmente en esos campos pujantes que Eagleton identificaba en 2012 como «a quartet of preoccupations: postcolonialism, ethnicity, sexuality and cultural studies» (ix). Basta tirar del hilo de un nombre, por caso Judith Butler, para haber acopiado en minutos una larga lista de comentarios, refutaciones, debates, papers, reseñas, entrevistas y libros sobre género y cultura, género y literatura, género y representaciones, género y narrativas, género y políticas y un largo y proliferante etcétera, sin que América Latina ni sus literaturas ni las lenguas en que se escribe aquí la crítica aparezcan concernidas sino a veces o entre tantos otros topónimos, corpus y situaciones. El afán teoricista (suponiendo que acordemos en qué sea eso) no es ni universal ni latinoamericano: distribuido de modo desparejo (lo que por otra parte puede decirse casi de cualquier cosa distribuible), el afán teoricista es no obstante global.

\section{Recuerdos de provincia}

Pero volvamos al vetusto panorama provinciano de "la teoría» que nos ocupa, es decir a la Argentina. Veamos cuánto resiste y para qué sirve mi simplificación —que aquí la teoría se enseña y se traduce, pero no se escribe- Se podría avanzar mencionando algunos casos. Los dos en que principalmente se apoya la simplificación son los de Enrique Pezzoni y Josefina Ludmer, que a mediados de los años 80 encabezaron los dos cursos de teoría literaria más importantes de la Universidad de Buenos Aires en los últimos 40 años y sin dudas muy influyentes en gran parte de la crítica y la enseñanza literaria en Argentina (como bien saben Analía Gerbaudo y otros especialistas, la impronta de esa enseñanza se lee aun hoy en los estilos de muchísimos profesores y en los manuales editados, los programas y las clases de literatura en las escuelas especialmente secundarias o del llamado nivel medio). ${ }^{4}$ Pezzoni publicó poco, pero casi por completo sobre temas de literatura argentina. El caso de Ludmer es diferente: aunque pueda decirse que Onetti es (como Horacio Quiroga o Felisberto Hernández) casi argentino, Ludmer podría haber sido identificada a principios de los 80 como una especialista en literatura latinoamericana: se la ubicaba por su libro sobre Cien años de soledad (1972) y por su más reciente Onetti. Los procesos de construcción del relato de 1977, aunque también como parte del grupo de la revista Literal, sin dudas un capítulo central de la teoría como pasión argentina. ${ }^{5}$ Sin embargo, los dos frondosos libros que Ludmer publicó durante la década en que protagonizó la renovación de la teoría literaria en el país, no están dedicados a desarrollar temas teóricos, sino a algunos de los grandes tópicos, libros y autores de la literatura nacional: el primero y más importante respecto de estas notas debido al momento en que se publica, su Tratado sobre el género gauchesco, de 1988; y en 1999, El cuerpo del delito, escrito en Yale mientras era profesora de literatura latinoamericana, pero enteramente dedicado también a literatura del Río de la Plata.

Podría parecer que el caso de Noé Jitrik (para mencionar otro eminente) arruinaría la hipótesis, porque antes y durante su prolongada carrera como profesor de literatura latinoamericana, publicó algunos textos que podemos tomar por teóricos (o más teóricos que críticos o que historiográficos); sin embargo, cualquiera tendría serias dificultades para aseverar que no se trata clara y principalmente de uno de los críticos e historiadores de literatura argentina más citados y prolíficos: cuando comenzó a dirigir la ambiciosa Historia crítica de la literatura argentina, Jitrik ya había 
escrito, por décadas, numerosos ensayos reconocidos y extensamente citados sobre ese campo. Ahora bien: ¿cuál sería, en cambio, el aporte teórico de Jitrik comparable en importancia, alcances o impacto en la investigación y en la enseñanza, con sus contribuciones a los estudios sobre Sarmiento, Hernández, la generación del 8o, Lugones, Quiroga, Macedonio, Cortázar? ¿Cuántos de sus lectores han leído la obra de Jitrik principalmente como teoría de la literatura?

Los escritos de Jorge Panesi sobre problemas teóricos y sobre aspectos de la obra de Derrida son insoslayables, pero la palabra clave de su trayectoria es "crítica»: Panesi es sobre todo un crítico de la crítica literaria argentina, de sus polémicas y sus relaciones con lo político y la política, y un estudioso de figuras y obras como las de Puig, Felisberto Hernández, Borges, Onetti, Perlongher, Kamenszain.

Es innegable que Alberto Giordano -el blanchotiano argentino más persistente y destacado en muchos años, aunque no escriba sobre Blanchot- ha hecho contribuciones insoslayables a las teorías de lo autobiográfico, de las escrituras del yo, a las teorías del ensayo y a los estudios barthesianos. Con todo, es difícil decidir si esas teorizaciones lo caracterizan más (o son de mayor importancia y valor crítico) que su texto sobre Saer —uno de los tres ensayos saerianos más importantes que se hayan escrito-, su libro sobre Puig, sus estudios sobre Felisberto, Arlt, Borges, Bioy, Bianco, Masotta, Bianciotti, María Moreno.

Se podría proseguir con muchos otros casos, pero estos ya alcanzan para dejar planteados los términos de una discusión posible.

\section{La erudición de las masas}

Desde hace algo más de dos siglos se ha ido acrecentando el papel decisivo que, junto con otras determinaciones principales, ha jugado eso que el siglo XX terminaría por llamar teoría literaria, en la emergencia, la formación y las transformaciones de dialectos y hablas culturales de poblaciones amplias de letrados, es decir de alfabetizados cada vez más numerosos que intervienen en conversaciones, intercambios, debates y producciones verbales, semióticas e imaginarias que dan por convencionalizadas y socialmente relevantes. En los espacios más o menos compartidos, desde los remanentes de la «esfera pública» y los media hasta las redes sociales, pasando por la escuela, hace tiempo no es difícil encontrar huellas, resonancias y fragmentos de la teoría. Sin esa especie de transglosia (en cuya composición no interviene solo la teoría, pero que no parece pensable sin ella), somos muchos y muy variados los sujetos que no podemos ni sabemos hablar de política, cultura, educación, literatura, artes, industrias del espectáculo y del entretenimiento, información y medios, mercados editoriales, historia y memoria.

Estamos transitando una época en que la teoría pudo haber perdido por completo el prestigio cientificista que le prestaba la vieja fe epistemológica de la modernidad; pero, a la inversa, la teoría parece haber acrecentado su pregnancia en los intercambios culturales; no es ya —según tanto se ha insistido - la teoría literaria stricto sensu de lo que hablamos, sino la teoría sin atributos, los estudios culturales en general, las filosofías de la subjetividad, etc. Como sea, si hace cuatro décadas yo hubiese utilizado la palabra «intertextualidad» en un programa radial sobre literatura, sus productores me hubiesen considerado un pedante academicista y no me hubiesen convocado ya más para hablar de libros al aire. Hoy la pronuncian de corrido y sin titubeo alguno, comentaristas de cine, teatro, artes, vida urbana, música o medios. Por aquellos años, cualquier editor hubiese considerado una extravagancia inapropiada que el relator de fútbol profesional de 
primera división más prestigioso y polémico del Cono Sur titulase Textualidades uno de sus libros (Morales). Algo parecido podría decirse de términos como "canon", "polifonía», "carnavalización", "campo intelectual», "capital simbólico», pero sin dudas el viaje más vertiginoso desde el neologismo jergal híper especializado hasta la conversación cultural cotidiana y de alcances masivos lo hizo la «deconstrucción», incluso en ámbitos lisa y llanamente populares. Son síntomas o fragmentos, en todo caso, de un proceso más vasto que Stuart Hall supo prever con mucha anticipación. A la luz de lo que ha sucedido en la Argentina en las últimas décadas, me interesa señalar dos factores que es necesario tener en cuenta aunque están lejos de agotar las determinaciones y contingencias que es preciso observar para entender las relaciones actuales entre «teoría» y vida cotidiana; son dos factores conectados: por un lado, el crecimiento de lo que podríamos llamar la estelarización antes solo periodística, luego televisiva y ahora sobre todo digital, de carismáticas figuras intelectuales como las de Noam Chomsky entre las menos novedosas y Slavoj Žižek entre las más recientes (y de otras menos televisables pero de una máxima originalidad y un atrevimiento teórico y político envidiable y alentador, como es el caso de Judith Butler). Y al mismo tiempo, el impacto de las políticas escolares y universitarias de los gobiernos latinoamericanos de la primera década del siglo usualmente estigmatizados como populistas (en Venezuela, Brasil, Bolivia, Ecuador, Uruguay y especialmente en Argentina). Los cursos de comunicación y de ciencias sociales y humanas de las universidades públicas de esos países no solo se multiplicaron: en el caso de la Argentina particularmente, llegaron a sectores sociales antes completamente ajenos a la enseñanza universitaria, e hicieron que miles de hijas e hijos de trabajadores tomaran clases que les permitían no sólo conocer aunque fuese por fotocopias y aunque fuera solo los rudimentos de las nociones laclausianas de populismo y hegemonía, sino hasta reconocer al autor de esas ideas cuando lo veían en la televisión pública o en YouTube conversando con Jacques Rancière, con Etienne Balibar o con Gianni Vattimo.

\section{Y cuanto yo escribir de vos deseo}

Algún lector de Yuri Tinianov podría argüir que entonces, si pasó a la vida cotidiana, la teoría se des-diferenció y ya no es tal. Y no obstante, conceder que la teoría esté muerta no parece fácil, desde que sigue dejando un tendal de apasionados cultores incondicionales, no solamente ardorosos sino, además, nuevos, y dedicados a líneas y preguntas teóricas también recientes y novedosas. ¿Cómo corroborar y sobre todo cómo interrogar esa persistencia reciente y presente?

A mediados de 2018 se publicó en Granada el primer número de Theory Now. Journal of Literature, Critique, and Thought. El volumen se abre con un monográfico de cinco textos dedicados a Jonathan Culler, uno de los integrantes del Comité Asesor de la publicación («Jonathan Culler: Theory now and again»), y comienza con un trabajo del propio Culler. Su estrategia para encarar el problema de la revista es paradójica, irónica y amable (una maestría que ya le conocíamos). En publicaciones recientes sobre teoría parece haber quedado atrás la insistencia de fines del siglo XX en dar por pasada, liquidada o museificada la teoría (aunque Terry Eagleton seguía con el asunto ya avanzada la segunda década de este siglo); por el contrario, argumentar que la teoría no está muerta —observa Culler- "parece haberse convertido en una industria en crecimiento», como lo demuestra la proliferación de títulos como After Theory, Theory after Theory, o Literary criticism in the 21st Century: Theory Renaissance. Culler hace un recorrido crítico informado y convincente por algunas de las principales líneas recientes de trabajo teórico y por sus tópicos y agendas más o menos innovadores, pero 
lo que me interesa subrayar aquí es la palabra clave con la que explica qué sigue impulsándonos a teorizar. La palabra, que Culler repite cuatro veces en las diez últimas líneas de su artículo, es deseo. La teoría es, por supuesto, un asunto de instituciones y de géneros discursivos, de escuelas y de ismos, de legados y tradiciones filosóficas, de políticas universitarias historizables y de compartimentación de los saberes. La teoría es, obviamente, un corpus de estudios, de ensayos y de libros publicados. Pero al mismo tiempo, «teoría» es la palabra que el siglo XX usó para nombrar un deseo, un deseo que no parece suprimible por los mismos motivos que provocan la declinación de disciplinas o la disminución de actividades relativas al saber y a sus políticas. Fiel a una teoría acerca de la teoría que viene formulando por lo menos desde $1997,{ }^{6}$ Culler describe ese deseo como un deseo del pensamiento acerca de sí mismo, un deseo de pensar acerca del pensar, con una resonancia remota pero audible de la tesis demaniana de «la resistencia a la teoría», aunque implicada de modo más explícito en lo que es dable ver como una perspectiva franca y activamente política.

Since theory not just an evolving corpus of works, but thinking about thinking, it calls us to question how a discipline frames questions, asking whether there are not other, better ways to proceed, and what we would mean by «better». The impetus to theory is a desire to understand what one is doing, to question commitments and their implications. Theory is driven by the impossible desire to step outside one's thought, both to place it and to understand it, and also by a desire -a possible desire- for change, both in the ways of one's own thought, which always could be sharper, more knowledgeable and capacious, more self-reflecting, and for change in the world which our thought engages, so there will always be new developments, will always be changes in the realm of theory, for a publication devoted to «theory today». (14)

Creo que tras la sospecha en que supimos ponerla junto a otras palabras largamente ocupadas por las lenguas de la dominación (disciplinas, filosofía, literatura...), «teoría» conserva sin embargo su utilidad: nos permite nombrar las escrituras que se efectúan por ese impulso, ese ímpetu, ese deseo del que habla Culler (por supuesto, la palabra es reemplazable: basta con que alguien encuentre una más útil y menos sobrecargada, una tarea que no se cuenta entre mis intereses). Creo que mientras tanto, seguimos usándola para señalar, de modo siempre tentativo, un tipo de pensamiento escrito - conjetural, especulativo y paradójico - acerca de ciertas contingencias donde interviene algo que seguimos llamando poesía, lectura, e incluso «literatura». Si eso es así, pierden interés asuntos como el tiempo de sobrevida con que cuente la crítica literaria, o si la crítica argentina escribe teoría o más bien — como parece haber sido su apasionada costumbre— la lee, la traduce, la enseña, la asedia y la usa con fidelidad y dedicación intensa. En este sentido, me cuento entre los profesores argentinos que en estos años hemos leído en el apasionamiento duradero de la crítica literaria por la teoría, un deseo del pensar impulsado - por supuesto- por un deseo de literatura, es decir por el acontecimiento que se resiste a la lectura, a la formalización atascada y no obstante siempre perseguida, a la teorización imposible y sin embargo permanentemente anhelada. Estoy seguro de que, con variaciones tonales o énfasis diversos, esta perspectiva reúne hoy el trabajo y la mirada de numerosos críticos argentinos profesores de literatura (pienso en los trabajos que en las últimas dos décadas han publicado, entre otros, Jorge Panesi, Jorge Monteleone, Alberto Giordano, Ana Porrúa, Daniel Link, Nora Avaro, Sandra Contreras). La siguiente definición de la teoría literaria, que sintetiza esa perspectiva, podría haber sido tomada de un escrito de Culler, pero la compuso Judith Podlubne, y creo que nos representa: 
dado que la literatura se define como el «cuestionamiento infinito de sí misma», la teoría surge con ella, no para contribuir a alcanzar una respuesta que ponga fin al planteo de la pregunta, sino para prolongar su diferimiento (...) Aunque el momento de institucionalización es inevitable, la teoría literaria no es una disciplina entre otras. La actividad teórica designa la tarea de conceptualización común a las disciplinas científicas, académicas, pero no se confunde ni equipara con ellas. El rasgo diferencial del auténtico saber teórico es la experimentación con el tenor performativo del concepto, el encuentro con el punto en que ese tenor excede la convención y vuelve infinitas sus posibilidades de significar. La autenticidad deriva de la fuerza con que la teoría se resiste a desconocer lo que sabe, su falta originaria, aun sabiendo que ese propósito está destinado al fracaso. (87-88)

\section{Notas}

1 Este trabajo retoma en parte, en parte reordena, algunas ideas y problemas que plantean trabajos recientes de Jonathan Culler, Graciela Montaldo y Judith Podlubne. Las dos últimas han discutido estos temas en esta revista, de modo que debo sumar a Analía Gerbaudo como estratega paciente y laboriosa y como anfitriona del debate reciente sobre la teoría literaria en el circuito universitario argentino. También prosigo desarrollos de algunos argumentos que propuse en trabajos anteriores, especialmente en The critical resistance of literature (2018) y en Resistencias a la lectura y resistencias a la teoría (2015).

2 No puedo extenderme en una situación no obstante bien pertinente para el tema de estas notas: no son pocas las revistas académicas argentinas que se presentan como especializadas en teoría literaria y que en realidad publican mayormente papers sobre temas de historia crítica de las literaturas y las culturas de América Latina: Orbis Tertius. Revista de teoría y crítica literaria (La Plata), El taco en la brea (Santa Fe), Estudios de teoría literaria (Mar del Plata); tal vez la revista Luthor. Entender, destruiry crear(Buenos Aires) sea una excepción y merezca una consideración aparte.

3 Me parece que no conviene exagerar el aislamiento no obstante característico de las comunidades académicas (su politicidad autoinstituida, digamos) respecto de la participación en las políticas ajenas a las universidades (es decir casi todo el campo de lo político), pero tampoco ignorar que en muchos casos — como en el del feminismo - el pasaje es multidireccional y las disimetrías no dislocan siempre para el mismo lado: a veces predomina la iniciativa de los movimientos emancipatorios hacia la teoría (y no viceversa). Insistiría, en cambio, en ese carácter auto-instituido y autónomo de la supuesta politicidad de la crítica cultural, debido a que carece casi por completo de la mirada examinadora y demandante permanente de los grupos políticos de otro tenor (como sabe todo el mundo, un líder territorial, un sindicalista o un legislador, por decir, no puede sostener su legitimidad únicamente en la mirada de sus pares sino a riesgo de perderla).

4 Inevitablemente, este asunto tiene algún aspecto autobiográfico: muchos de los profesores de teoría literaria y de literatura argentina de mi generación — los que en la segunda década del siglo XXI dirigimos las cátedras de las carreras de Letras del país- estudiamos en las clases o con los apuntes y los escritos de Pezzoni, de Ludmer y de otros que, como ellos, tenían entre 20 y 30 años de edad más que nosotros. Cuando alguien como uno habla de estos temas, actúa a la vez como investigador y como informante, a veces testigo de primera mano (lo que, como se sabe, no es siempre una ventaja y puede ser lo contrario).

5 La pasión por la teoría es una figura recientemente destacada por la investigación de Diego Peller, desde el título de su libro Pasiones teóricas. Crítica y literatura en los setenta de 2016. En 1994 y acerca del mismo tema, Daniel Link había usado con ironía un título célebre de Eduardo Mallea en su artículo «Historia de una pasión argentina. La crítica literaria 1955-1966».

6 Véanse Literary Theory. A Very Short Introduction (1997); The Literary in Theory (2007); y «Afterword: Theory Now and Again» (2011). 


\section{Referencias bibliográficas}

Contreras, S. (2002). Las vueltas de César Aira. Buenos Aires: Beatriz Viterbo.

Culler, J. (1997). Literary Theory. A Very Short Introduction. Oxford: Oxford University Press.

- (2007). The Literary in Theory. Stanford: Stanford University Press.

(2011). Afterword: Theory Now and Again. The South Atlantic Quarterly, 110(1), 223-230.

(2018). Critical Theory Today. Theory Now: Journal of literature, critique and thought, 1(1). http:// revistaseug.ugr.es/index.php/theorynow/article/view/7605/6802

Dalmaroni, M. (2015). Resistencias a la lectura y resistencias a la teoría. Algunos episodios en la crítica literaria latinoamericana. $452^{\circ}$ fahrenheit/revista de teoría de la literatura y literatura comparada, (12), 42-62. file:///C:/Users/Miguel/Downloads/290916-402967-1-SM\%2O(2).pdf

(2018). The critical resistance of literature (theoretical issues and Latin American discussions). Theory now: Journal of literature, critique and thought, 1(1). http://revistaseug.ugr.es/index.php/theorynow/ article/view/7600/6809

Eagleton, T. (2012). The Event of Literature. New Haven: Yale University Press.

Giordano, A. (1989). El efecto de irreal. Discusión, 1(1), 27-36. (1992). La experiencia narrativa. Juan José Saer, Felisberto Hernández, Manuel Puig. Rosario: Beatriz Viterbo.

(2001). Manuel Puig. La conversación infinita. Rosario: Beatriz Viterbo.

(2005). Modos del ensayo. De Borges a Piglia. Buenos Aires: Beatriz Viterbo.

Jitrik, N. (Dir.) (1999/2018). Historia crítica de la literatura argentina. 12 Tomos. Buenos Aires: Emecé.

Link, D. (1994). Historia de una pasión argentina. La crítica literaria 1955-1966. Cuadernos Hispanoamericanos, (527), 7-33.

Ludmer, J. (1972). Cien años de soledad. Una interpretación. Buenos Aires: Tiempo Contemporáneo. (1977). Onetti. Los procesos de construcción del relato. Buenos Aires: Sudamericana. (1988). El género gauchesco. Un tratado sobre la patria. Buenos Aires: Sudamericana. (1999). El cuerpo del delito. Un manual. Buenos Aires: Perfil Libros.

Montaldo, G. (2014). Teoría en fuga. El Taco en la brea, 1(1), 262-276. Recuperado de https://doi.org/10.14409/ tb.vii1.4216

Morales, V. H. (2017). Textualidades. La defensa de los sueños de siempre. Buenos Aires: Colihue.

Panesi, J. (1993). Felisberto Hernández. Rosario: Beatriz Viterbo.

- (2000). Críticas. Buenos Aires: Norma.

(2018). La seducción de los relatos. Crítica literaria y política en la Argentina. Buenos Aires: Eterna Cadencia.

Pezzoni, E. (1986). El texto y sus voces. Buenos Aires: Sudamericana.

Peller, D. (2016). Pasiones teóricas. Crítica y literatura en los setenta. Buenos Aires: Santiago Arcos.

Podlubne, J. (2017). Dossier: Fin y resistencia de la teoría. Presentación: La edad de la teoría literaria. El Taco en la brea, 15), 83-94. https://doi.org/10.14409/tb.vii5.6617

Sarlo, B. (1995). Borges. Un escritor en las orillas. Buenos Aires: Ariel. 